\title{
Forests of the Iguaçu National Park: Structure, Composition, and Richness
}

\author{
Ronan Felipe Souza $^{1}$ (D), Sebastião do Amaral Machado ${ }^{1}$ (D), Franklin Galvão ${ }^{1}$ (D), \\ Afonso Figueiredo Filho ${ }^{2}$ (D), Alex Costa Picoli ${ }^{3}$ \\ ${ }^{1}$ Universidade Federal do Paraná - UFPR, Curitiba/PR, Brasil \\ ${ }^{2}$ Universidade Estadual do Oeste do Paraná - UNICENTRO, Irati/PR, Brasil \\ ${ }^{3}$ In Natura Soluções Ambientais - IN, Curitiba/PR, Brasil
}

\begin{abstract}
Considering the importance of the Iguaçu National Park for the conservation of the Atlantic Forest and the absence of scientific or technical studies characterizing the ecology of forest species after seven and a half decades of its existence, a phytosociological survey of the arboreal vegetation was conducted to identify the various existing species and their successional stages. A total of 54 families, 135 genera, and 218 species were found in this survey. Euterpe edulis Mart. was the most frequently occurring species, which together with Aspidosperma polyneuron Müll. Arg., characterize the seasonal forests in the central and south regions of the park. In the north region, located $700 \mathrm{~m}$ asl, Araucaria angustifolia (Bertol.) Kuntze and Ilex paraguariensis A. St.-Hil. were observed along with some seasonal species, characterizing a transitional environment between seasonal and ombrophillous forests. In general, forests in the park were classified in advanced stages of ecological succession.
\end{abstract}

Keywords: successional stages, ecotone, semi-deciduous forests. 


\section{INTRODUCTION}

Aiming to avoid the complete deterioration of the Atlantic Forest Biome, laws have been enacted to ensure that degraded areas are recovered and the use of the remaining areas on farms is managed rationally. In addition, several protected units (UC) such as the Iguaçu National Park (INP) have been created.

Despite the effectiveness of surveillance and protection within the boundaries of the INP, numerous farms, residences, and sawmills had already been established there before the Park was created, mainly in its southwest region, where the vegetation was completely cleared for agriculture and livestock uses. In other areas, there was selective logging, leading to virtual disappearance of some of the prevailing species and reduction of the potential for natural regeneration in some places due to loss of the seed bank (Ferreira, 1999).

In this context, the Park's first management plan of this UC called for detailed studies of the floristic structure and phytosociological and ecological successional stages of the vegetation in different regions. According to Ziller (1998), these studies would establish the structural patterns of vegetation and species occurrences, which would direct the management and recovery in areas where natural succession had been compromised.

In characterizing the structure of a forest, the number of trees and species distribution are directly associated with the growth habits of the species and environmental conditions of the site (Lin et al., 2013). The assessment of parameters of horizontal and vertical structure must also be observed in characterizing the structure, as well as the percentages of importance and coverage (Mueller-Dambois \& Ellenberg, 1974).

After characterization of a particular forest area is performed, Meira \& Martins (2002) advised that the comparative floristic aspect should be emphasized, wherein different remnants could have their floral compositions confronted or related by similarity index (Ríos et al., 2010) or analysis grouping (Avila et al., 2011). Meira \& Martins (2002) also mentioned that such methods enable observation of the floristic proximity between different forest formations, which is useful to the understanding of the Brazilian forest phytogeography.

The importance of the INP for the conservation of forest species in the Atlantic Forest and the absence of technical information after seven and a half decades of its existence substantiate this study, which was conducted in order to identify the forest species and succession stages of the different existing vegetation formations.

\section{MATERIAL AND METHODS}

Study area - The Iguaçu National Park (INP) is located in the western region of the state of Paraná and encompasses a total area of $185,262.50$ hectares (ha). The geographic region occupied by the INP is characterized by Cfa climate (Alvares et al., 2013). The terrain is determined by the Iguaçu River watershed and lies between 100 and $750 \mathrm{~m}$ asl as from the river bank. Bhering (2007) published the latest soil classification conducted in Parana state; for the region of the INP, the following classes have been identified: Ortic Rendzic Chernosol, Haplic Gleysol, Eutrophic Litholic Neosol, Red Disferric Latosol, Eutrophic Red Latosol, and Red Eutroferric Nitosol, with predominance of Nitosol and Latosol.

Forests in the INP are composed of different vegetation formations. Alluvial, Submontane and Montane formations of Semi-deciduous Forest (FES) predominate in the south and central regions and, in the north region, an ecotone between FES and Ombrophillous Mixed Forest (FOM), as well as Alluvial FOM are observed (Souza et al., 2017).

Data and analysis - Seven groups of three plots were installed along the existing altitudinal gradient in the region from the Iguaçu River bank to the northernmost region of the Park. The plots were installed at intervals of $100 \mathrm{~m}$ asl in the West-East direction (Figure 1). In total, 21 permanent plots were installed, each sampling plot comprising an area of $2,000 \mathrm{~m}^{2}(20 \times 100 \mathrm{~m})$ totaling 4.20 ha.

Plot groups consisted of three plots: group one consisted of plots 1,2, and 3; group two was composed of plots 4, 5, and 6; and so on. Finally, group seven included plots 19,20, and 21. At each elevation, plots were positioned at variable distances from each other and parallel to the river course. They were distributed along the drainage slopes from their base up to the plateau regions near the watershed boundaries.

All living trees with circumference $\geq 15.70 \mathrm{~cm}$ $(\mathrm{DBH} \geq 5.00 \mathrm{~cm})$ were included in the survey and their 


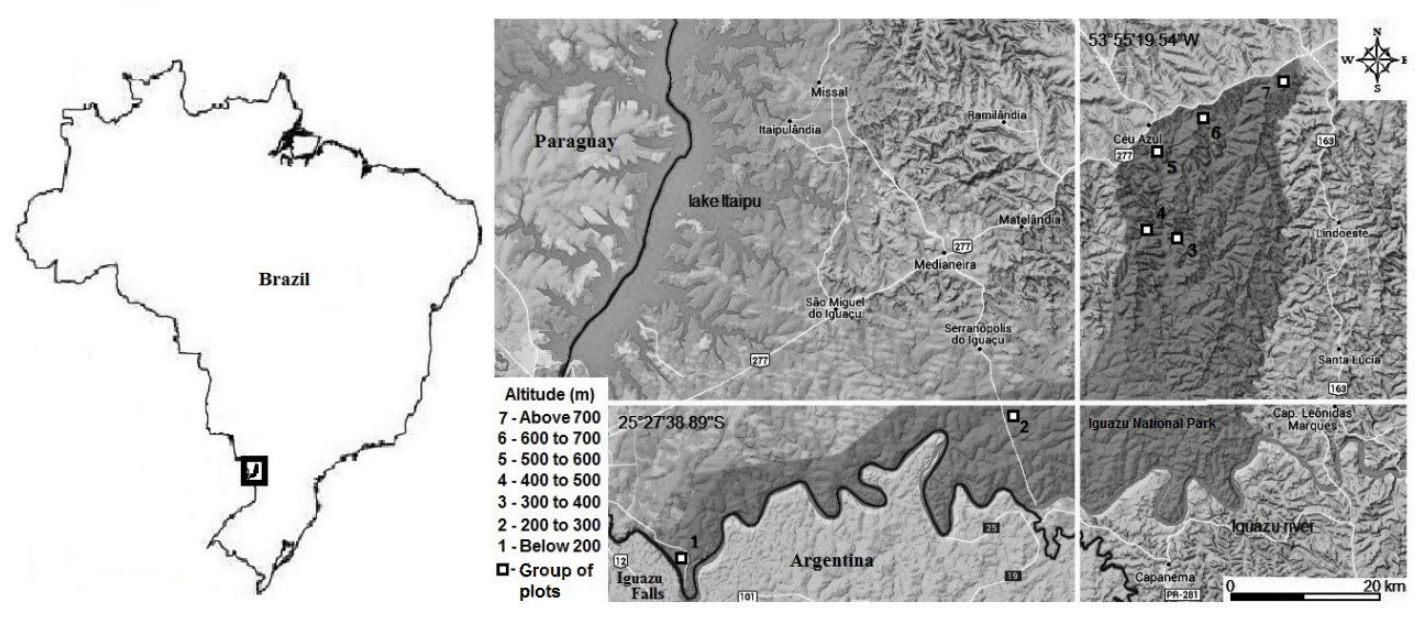

Figure 1. Localization of seven groups of plots installed in the Iguaçu National Park.

respective dendrologic materials were sent to the Botanical Museum of Curitiba for identification. The names were determined through a database search of the Missouri Botanical Garden (tropicos.org). Family classification followed the APG III (2009). Species were classified into Pioneer (PI), Light-Demanding Climax (CL), and Shade-Tolerant Climax (CS) according to adaptation from Oliveira-Filho et al. (1994) to the system proposed by Swaine \& Whitmore (1988), and considering the bibliographies of Ziller (1998), Jarenkow \& Waechter (2001), Silva et al. (2008), Gasper et al. (2013a), and Gasper et al. (2013b), as well as to field observations. The species were also classified by vegetation formation based on the analysis of the distribution records of species available at SpeciesLink (splink.org.br).

Vegetation sampling was conducted to ensure the observation of environmental changes in the INP, stratified into two levels so that all plots were installed in different environments. Even with the adoption of this sampling criterion, in order to verify the efficiency of the survey in relation to its floristic scope, a species-area curve was constructed to enable observation of the relationship between the number of species and the cumulative sampling effort (Felfili et al., 2011).

Characterization of the horizontal structure was performed by plot, in which 10 diameter classes with amplitude of $10 \mathrm{~cm}$ from the minimum diameter considered were arbitrarily defined to avoid an excessive number of classes to be grouped as trees with diameter $\geq 95 \mathrm{~cm}$. To characterize the vertical structure, heights from the ground to the morphological inversion point of trees were measured using a retractable graduated rod, and were then distributed into 11 height classes with amplitude of $2 \mathrm{~m}$ from the ground surface.

Plots were classified into three succession stages: initial, intermediate, and advanced, according to the following attributes: species richness (S); dominance (DOA) $\left(\mathrm{m}^{2} \cdot \mathrm{ha}^{-1}\right)$, density (DE) (trees.ha- $\left.{ }^{-1}\right)$, and cover value (CV) for the ecological groups; horizontal and vertical structure of vegetation. Decisions were also subsidized by contributions reported by Whitmore (1989), Schorn \& Galvão (2009), Holz et al. (2009), and Gasper et al. (2013b). The CONAMA resolution no. 2 of 18 March 1994 (Brasil, 1994) was observed for the ecological succession analysis despite not having been applied as a criterion for decisions.

Cover value for each ecological group was calculated by the following equation: $\mathrm{CV}=\mathrm{DR}+\mathrm{DOA}$, where: DR refers to the ratio between the density obtained for the ecological group and the total density observed in the plot; DOA refers to the ratio between the dominance of each ecological group and the total dominance observed in the plot.

Aiming at a good floristic characterization of the forest, the tree species observed by Ziller (1998) during a Rapid Ecological Assessment of the INP were added to the list. In this floristic survey, Ziller (1998) visited observation points distributed throughout the Park. Likewise, as before, all botanical material was sent to the Botanical Museum of Curitiba for identification. 


\section{RESULTS}

Floristic cover - In 10 plots, it was possible to sample 151 species, or $90 \%$ of the total. The remaining 11 plots contributed little to the increase in the number of species sampled, with addition of only 16 species, indicating that a large number of species occurred in common between the plots. In 20 plots, $100 \%$ of the species had already been sampled.
Floristic composition - Sampling of the plots showed occurrence of 4,299 trees that, when added to the species found by Ziller (1998), represented 54 botanical families, 135 genera, and 218 species (Table 1). Two trees measured in the plots could only be identified at the family level, which were grouped and assigned to the family Myrtaceae. Another nine trees could not be identified due to absence of leaves caused by seasonality, and were assigned to the "Unknown"

Table 1. Floristic checklist of the tree species in the Iguaçu National Park with their classification in ecological groups (GE), vegetation formation, occurrence in plot groups, and registry by similarity to voucher specimens deposited in the Botanic Museum of Curitiba (MBM).

$\begin{array}{ccccc}\text { Family/Species } & \text { GE } & \begin{array}{c}\text { Vegetation } \\ \text { Formation }\end{array} & \begin{array}{c}\text { Occurrence } \\ \text { in Plot } \\ \text { Groups }\end{array} & \begin{array}{c}\text { Voucher in } \\ \text { the MBM }\end{array}\end{array}$

\begin{tabular}{|c|c|c|c|c|c|}
\hline \multicolumn{6}{|l|}{ ANACARDIACEAE } \\
\hline Astronium graveolens Jacq. & $\mathrm{CL}$ & FES & FOM & 3.5 .7 & 7412 \\
\hline Lithraea brasiliensis March. & $\mathrm{CL}$ & - & FOM & - & - \\
\hline Mangifera indica $\mathrm{L}^{*}$ & $\mathrm{NC}$ & - & - & - & - \\
\hline Schinus therebinthifolia Raddi. & PI & FES & FOM & - & - \\
\hline Toxicodendron striatum (Ruiz \& Pav.) Kuntze & $\mathrm{NC}$ & - & - & 6 & 83904 \\
\hline \multicolumn{6}{|l|}{ ANNONACEAE } \\
\hline Annona cacans Warm. & CS & FES & - & 6 & 359684 \\
\hline Annona emarginata (Schltdl.) H. Rainer & $\mathrm{CL}$ & FES & FOM & 1.2.3.4.5.7 & 136781 \\
\hline Rollinia salicifolia Schltdl. & CS & FES & FOM & - & - \\
\hline \multicolumn{6}{|l|}{ APOCYNACEAE } \\
\hline Aspidosperma australe Müll. Arg. & CS & FES & - & 4 & 277233 \\
\hline Aspidosperma cylindrocarpon Müll. Arg. & CS & FES & - & - & - \\
\hline Aspidosperma polyneuron Müll. Arg. & CS & FES & - & 2.4.5.6.7 & 6773 \\
\hline Rauvolfia sellowii Müll. Arg. & $\mathrm{CL}$ & FES & - & 4.5 .6 & 69630 \\
\hline Tabernaemontana catharinensis A. DC. & PI & FES & - & 1.5 .7 & 36274 \\
\hline \multicolumn{6}{|l|}{ ASTERACEAE } \\
\hline Piptocarpha angustifolia Dusén ex Malme & PI & FES & FOM & - & - \\
\hline Vernonia discolor (Spreng.) Less. & PI & - & FOM & - & - \\
\hline \multicolumn{6}{|l|}{ AQUIFOLIACEAE } \\
\hline Ilex brevicuspis Reissek & $\mathrm{CL}$ & FES & FOM & 3.5 .7 & 16311 \\
\hline Ilex dumosa Reissek & $\mathrm{CL}$ & FES & FOM & 2 & 27053 \\
\hline Ilex paraguariensis A. St.-Hil. & CS & FES & FOM & 7 & 18976 \\
\hline Ilex theezans Mart. Ex Reissek & CS & - & FOM & - & - \\
\hline \multicolumn{6}{|l|}{ ARALIACEAE } \\
\hline Aralia warmingiana (Marchal) J. Wen & CS & FES & - & 6 & 157192 \\
\hline Schefflera morototoni (Aubl.) Maguire. Steyerm. \& Frodin & $\mathrm{CL}$ & FES & FOM & 4.6 & 12268 \\
\hline \multicolumn{6}{|l|}{ ARAUCARIACEAE } \\
\hline Araucaria angustifolia (Bertol.) Kuntze & $\mathrm{CL}$ & FES & FOM & 7 & 22489 \\
\hline \multicolumn{6}{|l|}{ ARECACEAE } \\
\hline Euterpe edulis Mart. & CS & FES & - & 1.2.4.5.6 & 9399 \\
\hline Syagrus romanzoffiana (Cham.) Glassman & PI & FES & FOM & 1.2.3.4.5.6.7 & 66225 \\
\hline
\end{tabular}

${ }^{*}$ Exotic plants; PI - Pioneer; CL - Light-Demanding Climax; CS - Shade-Tolerant Climax; NC - Not classified; FES - Semi-deciduous Forest; FOM - Ombrophillous Mixed Forest. The species exclusively observed by Ziller (1998) do not provide information on occurrence in the plot groups and on the MBM registry. 
Table 1. Continued...

\begin{tabular}{|c|c|c|c|c|c|}
\hline \multirow{2}{*}{ ASPARAGACEAE } & \multirow[t]{2}{*}{ GE } & \multicolumn{2}{|c|}{$\begin{array}{l}\text { Vegetation } \\
\text { Formation }\end{array}$} & \multirow[t]{2}{*}{$\begin{array}{l}\text { Occurrence } \\
\text { in Plot } \\
\text { Groups }\end{array}$} & \multirow[t]{2}{*}{$\begin{array}{l}\text { Voucher in } \\
\text { the MBM }\end{array}$} \\
\hline & & & & & \\
\hline Cordyline spectabilis Kunth \& C.D. Bouché & $\mathrm{CL}$ & FES & FOM & 2 & 266966 \\
\hline \multicolumn{6}{|l|}{ BIGNONIACEAE } \\
\hline Handroanthus albus (Cham.) Mattos & $\mathrm{CL}$ & FES & FOM & 5 & 66524 \\
\hline Handroanthus chrysotrichus (Mart. ex A. DC.) Mattos. & $\mathrm{CL}$ & FES & - & - & - \\
\hline Handroanthus heptaphyllus (Vell.) Mattos & $\mathrm{CL}$ & FES & - & 3.5 .6 & 384364 \\
\hline Jacaranda micrantha Cham. & PI & FES & FOM & 1.3.5.6.7 & 11273 \\
\hline Jacaranda puberula Cham. & PI & - & FOM & 7 & 70749 \\
\hline \multicolumn{6}{|l|}{ BORAGINACEAE } \\
\hline Cordia americana (L.) Gottschling \& J. S. Mill. & CL & FES & - & 1.2.3.4.5 & 336335 \\
\hline Cordia ecalyculata Vell. & $\mathrm{CL}$ & FES & - & 1.2.4.5.6.7 & 236875 \\
\hline Cordia superba Cham. & CS & FES & - & 6 & 128764 \\
\hline Cordia trichotoma (Vell.) Arráb. Ex Steud. & $\mathrm{CL}$ & FES & - & 1.2.3.4.5.6.7 & 21646 \\
\hline \multicolumn{6}{|l|}{ CALOPHYLLACEAE } \\
\hline Calophyllum brasiliense Cambess. & CS & FES & - & 4 & 287625 \\
\hline \multicolumn{6}{|l|}{ CANELLACEAE } \\
\hline Capsicodendron dinisii (Schwacke) Occhioni & CL & - & FOM & - & - \\
\hline \multicolumn{6}{|l|}{ CANNABACEAE } \\
\hline Celtis iguanaea (Jacq.) Sarg. & PI & FES & FOM & 3 & 260947 \\
\hline Trema micrantha (L.) Blume & PI & FES & FOM & 6 & 63979 \\
\hline \multicolumn{6}{|l|}{ CARDIOPTERIDACEAE } \\
\hline Citronella gongonha (Mart.) R.A. Howard & $\mathrm{CL}$ & FES & - & 2.4 .5 & 136760 \\
\hline Citronella paniculata (Mart.) R.A. Howard & CL & FES & FOM & 5 & 4465 \\
\hline \multicolumn{6}{|l|}{ CARICACEAE } \\
\hline Jacaratia spinosa (Aubl.) A. DC. & CL & FES & - & 1.2.4.5.6.7 & 149157 \\
\hline \multicolumn{6}{|l|}{ CELASTRACEAE } \\
\hline Maytenus alaternoides Reissek & CS & FES & FOM & - & - \\
\hline Maytenus aquifolium Mart. & CS & FES & FOM & 2 & 72301 \\
\hline \multicolumn{6}{|l|}{ CLUSIACEAE } \\
\hline Garcinia gardneriana (Planch. \& Triana) Zappi & CS & FES & - & 4 & 342548 \\
\hline \multicolumn{6}{|l|}{ ERYTHROXYLACEAE } \\
\hline Erythroxylum deciduum A. St.-Hil. & CL & FES & FOM & 7 & 15662 \\
\hline \multicolumn{6}{|l|}{ EUPHORBIACEAE } \\
\hline Actinostemon concolor (Spreng.) Müll. Arg. & CS & FES & FOM & - & - \\
\hline Alchornea glandulosa Poepp. & $\mathrm{CL}$ & FES & FOM & 2.5.6.7 & 222764 \\
\hline Alchornea sidifolia Müll. Arg. & CS & FES & FOM & - & - \\
\hline Alchornea triplinervia (Spreng.) Müll. Arg. & CL & FES & FOM & 1.3.4.5.6.7 & 135940 \\
\hline Croton urucurana Baill. & PI & FES & - & - & - \\
\hline Sapium glandulatum (Vell.) Pax & PI & FES & FOM & - & - \\
\hline Sebastiania brasiliensis Spreng. & CS & FES & FOM & 2.3.4.5.6.7 & 255056 \\
\hline Sebastiania commersoniana (Baill.) L. B. Sm. \& Downs & CS & FES & FOM & 2.3.6.7 & 1572 \\
\hline $\begin{array}{l}\text { Sebastiania schottiana var. angustifolia (Müll. Arg.) Pax. \& } \\
\text { K. Hoffm }\end{array}$ & PI & FES & - & - & - \\
\hline \multicolumn{6}{|l|}{ FABACEAE } \\
\hline Acacia bimucronata DC. & PI & FES & - & 1.4 & 3064 \\
\hline Albizia edwallii (Hoehne) Barneby \& J.W. Grimes & PI & FES & FOM & 1 & 9859 \\
\hline
\end{tabular}

*Exotic plants; PI - Pioneer; CL - Light-Demanding Climax; CS - Shade-Tolerant Climax; NC - Not classified; FES - Semi-deciduous Forest; FOM - Ombrophillous Mixed Forest. The species exclusively observed by Ziller (1998) do not provide information on occurrence in the plot groups and on the MBM registry. 
Table 1. Continued...

\begin{tabular}{|c|c|c|c|c|c|}
\hline \multirow{2}{*}{$\begin{array}{c}\text { Family/Species } \\
\text { Albizia niopoides (Spuce ex Benth.) Burkart }\end{array}$} & \multirow{2}{*}{$\begin{array}{l}\text { GE } \\
\text { PI }\end{array}$} & \multicolumn{2}{|c|}{$\begin{array}{l}\text { Vegetation } \\
\text { Formation }\end{array}$} & \multirow{2}{*}{$\begin{array}{l}\text { Occurrence } \\
\text { in Plot } \\
\text { Groups } \\
1.2 .4\end{array}$} & \multirow{2}{*}{$\begin{array}{c}\text { Voucher in } \\
\text { the MBM } \\
78526\end{array}$} \\
\hline & & FES & - & & \\
\hline Anadenanthera colubrina (Vell.) Brenan & $\mathrm{CL}$ & FES & - & 2.4 & 201656 \\
\hline Apuleia leiocarpa (Vogel) J. F. Macbr. & $\mathrm{CL}$ & FES & - & 1.4.5.6 & 14941 \\
\hline Bauhinia forficata Link & PI & FES & FOM & 1.3.6 & 9120 \\
\hline Calliandra foliolosa Benth. & CS & FES & - & 3.4 .6 & 234521 \\
\hline Copaifera langsdorffii Desf. & $\mathrm{CL}$ & FES & - & - & - \\
\hline Dalbergia brasiliensis Vogel. & $\mathrm{CL}$ & FES & FOM & - & - \\
\hline Dalbergia frutescens (Vell.) Britton & $\mathrm{CL}$ & FES & FOM & 2.3.4.5.7 & 243562 \\
\hline Dalbergia sp. & NC & - & - & - & - \\
\hline Enterolobium contortisiliquum (Vell.) Morong & CL & FES & - & 2.3 & 9857 \\
\hline Erythrina falcata Benth. & $\mathrm{CL}$ & FES & FOM & 3.4 .7 & 70136 \\
\hline Holocalyx balansae Micheli & CS & FES & - & 1.2.3.4.5.6 & 12641 \\
\hline Inga marginata Willd. & $\mathrm{CL}$ & FES & FOM & 1.4.5.6 & 234522 \\
\hline Inga striata Benth. & $\mathrm{CL}$ & FES & FOM & 3.7 & 210008 \\
\hline Inga uruguensis Hook. \& Arn. & PI & FES & - & - & - \\
\hline Inga vera subsp. affinis (DC.) T.D. Penn. & $\mathrm{CL}$ & FES & FOM & 2.3.7 & 9255 \\
\hline Inga virescens Benth. & PI & FES & FOM & - & - \\
\hline Lonchocarpus campestris Mart. Ex Benth. & $\mathrm{CL}$ & FES & FOM & 1.2.3.4.5.6.7 & 8475 \\
\hline Lonchocarpus cultratus (Vell.) A.M.G. Azevedo \& H. C. Lima & $\mathrm{CL}$ & FES & FOM & 3 & 11747 \\
\hline Lonchocarpus leucanthus Burkart & $\mathrm{CL}$ & FES & FOM & 1.4 & - \\
\hline Lonchocarpus muehlbergianus Hassl. & $\mathrm{CL}$ & FES & FOM & 1 & 248961 \\
\hline Lonchocarpus nitidus (Vogel) Benth. & $\mathrm{CL}$ & FES & FOM & 1.3.4.5 & 40400 \\
\hline Machaerium paraguariense Hassl. & $\mathrm{CL}$ & FES & FOM & 1.3.4.5 & 345372 \\
\hline Machaerium stipitatum (DC.) Vogel & CL & FES & FOM & 1.2.3.4.5.6.7 & 63596 \\
\hline Myrocarpus frondosus Allemão & $\mathrm{CL}$ & FES & - & 2.4.5.6.7 & 218359 \\
\hline Myroxylon peruiferum L. f. & $\mathrm{CS}$ & FES & - & 2.3.5 & 1033 \\
\hline Parapiptadenia rigida (Benth.) Brenan & $\mathrm{CL}$ & FES & - & 1.2.3.4.5.6.7 & 14927 \\
\hline Peltophorum dubium (Spreng.) Taub. & $\mathrm{CL}$ & FES & - & 2.5.7 & 53529 \\
\hline Pterogyne nitens Tul. & $\mathrm{CL}$ & FES & - & - & - \\
\hline Schizolobium parahyba (Vell.) S.F. Blake & PI & FES & - & 6 & 348640 \\
\hline Senegalia polyphylla (DC.) Britton & PI & FES & - & 5 & 9867 \\
\hline Senegalia recurva (Benth.) Seigler \& Ebinger & PI & FES & FOM & 5.7 & 7015 \\
\hline Senegalia velutina (DC.) Seigler \& Ebinger & PI & FES & - & 2 & 9883 \\
\hline \multicolumn{6}{|l|}{ LAMIACEAE } \\
\hline Aegiphila mediterranea Vell. & PI & FES & FOM & 1.2.4.5 & 15014 \\
\hline Aegiphila sellowiana Cham. & PI & FES & FOM & 5 & 257076 \\
\hline Vitex megapotamica (Spreng.) Moldenke & $\mathrm{CL}$ & FES & FOM & 3 & 67566 \\
\hline \multicolumn{6}{|l|}{ LAURACEAE } \\
\hline Cinnamomum glaziovii (Mez) Kosterm. & $\mathrm{CS}$ & - & FOM & 6 & -- \\
\hline Cinnamomum sellowianum (Nees \& Mart.) Koesterm. & $\mathrm{CL}$ & - & FOM & 6.7 & 249663 \\
\hline Cryptocarya aschersoniana $\mathrm{Mez}$ & CS & FES & FOM & - & - \\
\hline Endlicheria paniculata (Spreng.) J. F. Macbr. & CS & FES & - & - & 234475 \\
\hline Nectandra grandiflora Nees \& mart. ex Nees & CS & FES & FOM & - & - \\
\hline Nectandra lanceolata Nees \& Mart. & CS & FES & FOM & 1.2.3.4.5.6.7 & 23258 \\
\hline Nectandra megapotamica (Spreng.) Mez. & CS & FES & FOM & 1.2.3.4.5.6.7 & 234482 \\
\hline Nectandra sp. & $\mathrm{NC}$ & - & - & - & - \\
\hline
\end{tabular}

${ }^{*}$ Exotic plants; PI - Pioneer; CL - Light-Demanding Climax; CS - Shade-Tolerant Climax; NC - Not classified; FES - Semi-deciduous Forest; FOM - Ombrophillous Mixed Forest. The species exclusively observed by Ziller (1998) do not provide information on occurrence in the plot groups and on the MBM registry. 
Table 1. Continued...

\begin{tabular}{|c|c|c|c|c|c|}
\hline \multirow{2}{*}{$\begin{array}{l}\text { Family/Species } \\
\text { Ocotea acutifolia (Nees) Mez }\end{array}$} & \multirow{2}{*}{$\begin{array}{l}\text { GE } \\
\text { CS }\end{array}$} & \multicolumn{2}{|c|}{$\begin{array}{l}\text { Vegetation } \\
\text { Formation }\end{array}$} & \multirow{2}{*}{$\begin{array}{l}\text { Occurrence } \\
\text { in Plot } \\
\text { Groups } \\
\text { - }\end{array}$} & \multirow{2}{*}{$\begin{array}{c}\text { Voucher in } \\
\text { the MBM } \\
-\end{array}$} \\
\hline & & FES & FOM & & \\
\hline Ocotea diospyrifolia (Meisn.) Mez. & CS & FES & FOM & 1.2.3.4.5.6.7 & 111187 \\
\hline Ocotea indecora (Schott) Mez. & $\mathrm{CS}$ & FES & FOM & 4.7 & 335848 \\
\hline Ocotea porosa (Nees \& Mart.) Barroso & CS & - & FOM & - & - \\
\hline Ocotea puberula (Rich.) Nees & $\mathrm{CL}$ & FES & FOM & 1.2.5.7 & 201053 \\
\hline Ocotea pulchella Mart. & CS & FES & FOM & - & - \\
\hline Ocotea silvestris Vattimo-Gil & CS & FES & FOM & 2.4.5.6.7 & 159701 \\
\hline \multicolumn{6}{|l|}{ LECYTHIDACEAE } \\
\hline Cariniana legalis (Mart.) Kuntze & $\mathrm{CL}$ & FES & - & - & - \\
\hline \multicolumn{6}{|l|}{ LOGANIACEAE } \\
\hline Strychnos brasiliensis (Spreng.) Mart. & $\mathrm{CL}$ & FES & FOM & 2.3.4.5.7 & 67211 \\
\hline \multicolumn{6}{|l|}{ MALVACEAE } \\
\hline Bastardiopsis densiflora (Hook. \& Arn.) Hassl. & $\mathrm{CL}$ & FES & - & 1.2.3.4.5 & 313106 \\
\hline Ceiba speciosa (A. St.-Hil.) Ravernna & $\mathrm{CL}$ & FES & - & 1.4 .5 & 359685 \\
\hline Guazuma ulmifolia Lam. & $\mathrm{CL}$ & FES & - & 7 & 239761 \\
\hline Heliocarpus popayanensis Kunth & PI & FES & - & 1 & 338069 \\
\hline Luehea divaricata Mart. & $\mathrm{CL}$ & FES & FOM & 1.3.4.7 & 66585 \\
\hline \multicolumn{6}{|l|}{ MELASTOMATACEAE } \\
\hline Miconia hymenonervia (Raddi) Cogn. & $\mathrm{CS}$ & FES & - & 4.5 .7 & - \\
\hline Miconia pusilliflora Beurl. & CS & FES & - & 7 & 7859 \\
\hline \multicolumn{6}{|l|}{ MELIACEAE } \\
\hline Cabralea canjerana (Vell.) Mart. & $\mathrm{CL}$ & FES & FOM & 1.2.3.4.5.6.7 & 66335 \\
\hline Cedrela fissilis Vell. & $\mathrm{CL}$ & FES & FOM & 1.2.3.4.5.6.7 & 54094 \\
\hline Guarea kunthiana A. Juss. & CS & FES & - & 1.4 .6 & 37543 \\
\hline Guarea macrophylla Vahl & CS & FES & FOM & 4.6 & 348527 \\
\hline Trichilia casaretti C. DC. & CS & FES & - & 4.5 .6 & 283080 \\
\hline Trichilia catigua A. Juss. & $\mathrm{CS}$ & FES & - & 1.2.3.4.6.7 & 8291 \\
\hline Trichilia claussenii C. DC. & CS & FES & FOM & 2.4.5.6 & 37522 \\
\hline Trichilia elegans A. Juss. & CS & FES & FOM & 1.2.3.4.5.6 & 103578 \\
\hline Trichilia pallens C. DC. & $\mathrm{CS}$ & FES & FOM & 1.7 & 37524 \\
\hline \multicolumn{6}{|l|}{ MONIMIACEAE } \\
\hline Hennecartia omphalandra J. Poiss. & CS & FES & FOM & 1.2.3.5.7 & 104156 \\
\hline Mollinedia blumenaviana Perkins & $\mathrm{CS}$ & - & FOM & 6 & 15039 \\
\hline Mollinedia clavigera Tul. & CS & - & FOM & 6.7 & 147658 \\
\hline \multicolumn{6}{|l|}{ MORACEAE } \\
\hline Ficus insipida Willd. & $\mathrm{CL}$ & FES & - & - & - \\
\hline Ficus luschnathiana (Miq.) Miq. & $\mathrm{CL}$ & FES & FOM & 2.4 .6 & 251016 \\
\hline Ficus sp. & NC & - & - & - & - \\
\hline Maclura tinctoria (L.) O. Don ex Steud & $\mathrm{CL}$ & FES & - & 1.5 .6 & 66415 \\
\hline Sorocea bonplandii (Baill.) W.C. Burger. et al. & CS & FES & FOM & 1.2.4.5.67 & 43753 \\
\hline \multicolumn{6}{|l|}{ MYRTACEAE } \\
\hline Calycorectes riedelianus $\mathrm{O}$. Berg & CS & FES & - & - & - \\
\hline Campomanesia guazumifolia (Cambess.) O. Berg & CS & FES & FOM & 1 & 47731 \\
\hline Campomanesia xanthocarpa Mart. Ex O. Berg. & CS & FES & FOM & 1.2.3.4.5.6.7 & 66536 \\
\hline Eucalyptus sp.* & NC & - & - & - & - \\
\hline Eugenia burkartiana (D. Legrand) D. Legrand & CS & FES & FOM & 1.2.4.6 & 6554 \\
\hline
\end{tabular}

${ }^{*}$ Exotic plants; PI - Pioneer; CL - Light-Demanding Climax; CS - Shade-Tolerant Climax; NC - Not classified; FES - Semi-deciduous Forest; FOM - Ombrophillous Mixed Forest. The species exclusively observed by Ziller (1998) do not provide information on occurrence in the plot groups and on the MBM registry. 
Table 1. Continued...

\begin{tabular}{|c|c|c|c|c|c|}
\hline \multirow{2}{*}{$\begin{array}{l}\text { Family/Species } \\
\text { Eugenia clorophylla O. Berg }\end{array}$} & \multirow{2}{*}{$\begin{array}{l}\text { GE } \\
\text { CS }\end{array}$} & \multicolumn{2}{|c|}{$\begin{array}{l}\text { Vegetation } \\
\text { Formation }\end{array}$} & \multirow{2}{*}{$\begin{array}{l}\text { Occurrence } \\
\text { in Plot } \\
\text { Groups } \\
4.7\end{array}$} & \multirow{2}{*}{$\begin{array}{c}\text { Voucher in } \\
\text { the MBM }\end{array}$} \\
\hline & & FES & FOM & & \\
\hline Eugenia hiemalis Cambess. & $\mathrm{CS}$ & FES & FOM & 2 & 391511 \\
\hline Eugenia involucrata DC. & $\mathrm{CL}$ & FES & FOM & 2.3 & 170424 \\
\hline Eugenia pyriformis Cambess. & $\mathrm{CL}$ & FES & FOM & 1.2.3.4.7 & 66537 \\
\hline Eugenia ramboi D. Legrand & $\mathrm{CS}$ & FES & FOM & 4.7 & 10842 \\
\hline Eugenia subterminalis DC. & $\mathrm{CL}$ & FES & - & 2.5 & - \\
\hline Myrcia laruotteana Cambess. & $\mathrm{CS}$ & FES & FOM & 2.5 & 238806 \\
\hline Myrcia rostrata DC. & $\mathrm{CS}$ & FES & FOM & - & - \\
\hline Myrciaria floribunda (H. West ex Willd.) O. Berg & $\mathrm{CL}$ & FES & FOM & 7 & 66217 \\
\hline Myrtaceae & $\mathrm{NC}$ & - & - & 5 & - \\
\hline Pimenta pseudocaryophyllus (Gomes) L. R. Landrum & $\mathrm{CL}$ & - & FOM & - & - \\
\hline Plinia rivularis (Cambess.) Rotman & $\mathrm{CS}$ & FES & - & 1.2.3.4.6 & 132200 \\
\hline Psidium cattleyanum Sabine & $\mathrm{CL}$ & - & FOM & - & - \\
\hline \multicolumn{6}{|l|}{ NYCTAGINACEAE } \\
\hline Neea schwackeana Heimerl & $\mathrm{CL}$ & FES & - & 2 & 250272 \\
\hline Pisonia ambigua Heimerl & $\mathrm{CL}$ & FES & - & 1.6 & 71877 \\
\hline \multicolumn{6}{|l|}{ OPILIACEAE } \\
\hline Agonandra engleri Hoehne & CL & FES & - & 7 & 235155 \\
\hline \multicolumn{6}{|l|}{ PINACEAE } \\
\hline Pinus sp.* & $\mathrm{NC}$ & - & - & - & - \\
\hline \multicolumn{6}{|l|}{ PHYTOLACCACEAE } \\
\hline Gallesia integrifolia (Spreng.) Harms & $\mathrm{CL}$ & FES & - & - & - \\
\hline Seguieria guaranitica Speg. & $\mathrm{CL}$ & FES & - & 1.2.3.4.5.6 & 52723 \\
\hline \multicolumn{6}{|l|}{ PIPERACEAE } \\
\hline Piper amalago L. & $\mathrm{CL}$ & FES & - & 1.4 & 191797 \\
\hline \multicolumn{6}{|l|}{ PODOCARPACEAE } \\
\hline Podocarpus lambertii Klotzsch ex Endl. & CS & FES & FOM & - & - \\
\hline \multicolumn{6}{|l|}{ POLYGONACEAE } \\
\hline Ruprechtia laxiflora Meisn. & CS & FES & - & 1.3.4.5 & 9262 \\
\hline \multicolumn{6}{|l|}{ PRIMULACEAE } \\
\hline Myrsine coriacea (Sw.) R. Br. Ex Roem. \& Schult. & PI & FES & FOM & 2.4 & 186138 \\
\hline Myrsine umbellata Mart. & PI & FES & FOM & 1.2.3.4.5.7 & 186139 \\
\hline \multicolumn{6}{|l|}{ PROTEACEAE } \\
\hline Grevillea robusta A. Cunn. Ex R. Br.* & $\mathrm{NC}$ & - & - & - & - \\
\hline Roupala asplenioides Sleumer & $\mathrm{CL}$ & FES & - & 5 & 10288 \\
\hline Roupala brasiliensis Klotzsch & $\mathrm{CL}$ & FES & FOM & 7 & 29157 \\
\hline \multicolumn{6}{|l|}{ RHAMNACEAE } \\
\hline Colubrina glandulosa Perkins & $\mathrm{CL}$ & FES & - & - & - \\
\hline Hovenia dulcis Thunb.* & $\mathrm{NC}$ & - & - & 6.7 & 26374 \\
\hline \multicolumn{6}{|l|}{ ROSACEAE } \\
\hline Prunus myrtifolia (L.) Urb. & $\mathrm{CL}$ & FES & FOM & 2.3.4.5.6.7 & 295 \\
\hline Prunus sellowii Koehne & $\mathrm{CL}$ & FES & FOM & - & - \\
\hline \multicolumn{6}{|l|}{ RUBIACEAE } \\
\hline Alseis floribunda Schott & CS & FES & - & 2 & 129304 \\
\hline Faramea cyanea Müll. Arg. & $\mathrm{CL}$ & FES & - & - & - \\
\hline Ixora velutina Wall. & $\mathrm{CS}$ & FES & - & 2.4.5.6 & - \\
\hline
\end{tabular}

${ }^{*}$ Exotic plants; PI - Pioneer; CL - Light-Demanding Climax; CS - Shade-Tolerant Climax; NC - Not classified; FES - Semi-deciduous Forest; FOM - Ombrophillous Mixed Forest. The species exclusively observed by Ziller (1998) do not provide information on occurrence in the plot groups and on the MBM registry. 
Table 1. Continued...

\begin{tabular}{|c|c|c|c|c|c|}
\hline \multirow{2}{*}{$\begin{array}{l}\text { Family/Species } \\
\text { Psychotria carthagenensis Jacq. }\end{array}$} & \multirow{2}{*}{$\begin{array}{l}\text { GE } \\
\text { CS }\end{array}$} & \multicolumn{2}{|c|}{$\begin{array}{l}\text { Vegetation } \\
\text { Formation }\end{array}$} & \multirow{2}{*}{$\begin{array}{l}\text { Occurrence } \\
\text { in Plot } \\
\text { Groups } \\
2.4 .6 .7\end{array}$} & \multirow{2}{*}{$\begin{array}{c}\begin{array}{c}\text { Voucher in } \\
\text { the MBM }\end{array} \\
238789\end{array}$} \\
\hline & & FES & FOM & & \\
\hline Rudgea jasminoides (Cham.) Müll. Arg. & CS & FES & FOM & 2.7 & 384884 \\
\hline Simira sampaioana (Standl.) Steyerm. & $\mathrm{CL}$ & FES & - & 1 & 12578 \\
\hline \multicolumn{6}{|l|}{ RUTACEAE } \\
\hline Balfourodendron riedelianum (Engl.) Engl. & CS & FES & - & 1.2.3.4.5.6 & 48772 \\
\hline Citrus limon(L.) Osbeck ${ }^{*}$ & NC & - & - & 7 & 35846 \\
\hline Citrus sinensis (L.) Osbek ${ }^{*}$ & NC & - & - & 1.2 .7 & 255839 \\
\hline Helietta apiculata Benth. & PI & FES & - & 2.3 & 8482 \\
\hline Pilocarpus pennatifolius Lem. & CS & FES & FOM & 1.3.4 & 103178 \\
\hline Zanthoxylum kleinii (R. S. Cowan) P. G. Waterman & PI & FES & FOM & - & - \\
\hline Zanthoxylum naranjillo Griseb. & PI & FES & - & 1.2.3.5 & 195738 \\
\hline Zanthoxylum petiolare A. St.-Hil. \& Tul. & PI & FES & - & 2.3.5 & 11779 \\
\hline Zanthoxylum rhoifolium Lam. & PI & FES & FOM & 2.3.4.5 & 17977 \\
\hline \multicolumn{6}{|l|}{ SALICACEAE } \\
\hline Banara tomentosa Clos & CS & FES & FOM & 2.3.6.7 & 38001 \\
\hline Casearia decandra Jacq. & CS & FES & FOM & 1.2.3.4.5.7 & 67198 \\
\hline Casearia lasiophylla Eichler & $\mathrm{CL}$ & FES & FOM & 7 & 348529 \\
\hline Casearia obliqua Spreng. & CS & FES & FOM & 1.2 .7 & 5067 \\
\hline Casearia sylvestris Sw. & $\mathrm{CL}$ & FES & FOM & 1.2.4.6.7 & 10832 \\
\hline Prockia crucis P. Browne ex L. & $\mathrm{CL}$ & FES & FOM & 3.4.5.6.7 & 135207 \\
\hline Xylosma ciliatifolia (Clos) Eichler & $\mathrm{CL}$ & FES & FOM & 7 & 4286 \\
\hline \multicolumn{6}{|l|}{ SAPINDACEAE } \\
\hline Allophylus edulis (A. St.-Hil.. et al.) Hieron. Ex Niederl. & $\mathrm{CL}$ & FES & FOM & 1.2.3.4.5.6.7 & 348531 \\
\hline Allophylus guaraniticus Radlk. & $\mathrm{CL}$ & FES & FOM & - & - \\
\hline Cupania vernalis Cambess. & $\mathrm{CL}$ & FES & FOM & 3.5.6.7 & 345421 \\
\hline Diatenopteryx sorbifolia Radlk. & $\mathrm{CL}$ & FES & - & 1.2.3.4.5.6.7 & 111280 \\
\hline Matayba elaeagnoides Radlk. & $\mathrm{CL}$ & FES & FOM & 3.5 .7 & 80089 \\
\hline \multicolumn{6}{|l|}{ SAPOTACEAE } \\
\hline $\begin{array}{l}\text { Chrysophyllum gonocarpum (Mart. \& Eichler ex Miq.) } \\
\text { Engl. }\end{array}$ & CS & FES & - & 1.2.3.4.5.6.7 & 174840 \\
\hline Chrysophyllum marginatum (Hook. \& Arn.) Radlk. & $\mathrm{CL}$ & FES & FOM & 1.2.3.4.5.6.7 & 239238 \\
\hline \multicolumn{6}{|l|}{ SIMAROUBACEAE } \\
\hline Picrasma crenata Engl. In Engl. \& Prantl & CS & FES & FOM & 2.3 & 130135 \\
\hline \multicolumn{6}{|l|}{ SOLANACEAE } \\
\hline Cestrum intermedium Sendtn. & CL & FES & FOM & 1.5 .6 & 15466 \\
\hline Cestrum strigilatum Ruiz \& Pav. & PI & FES & - & 5 & 3095 \\
\hline Solanum argenteum Dunal & PI & FES & - & 5 & 4246 \\
\hline Solanum campaniforme Roem. \& Schult. & PI & FES & - & 5 & 345427 \\
\hline Solanum granuloso-leprosum Dunal & PI & FES & FOM & 3.5 & 56670 \\
\hline Solanum guaraniticum A. St.-Hil. & PI & FES & FOM & 7 & 67654 \\
\hline Solanum mauritianum Scop. & PI & FES & FOM & 3 & 345423 \\
\hline Solanum pseudoquina A. St.-Hil. & PI & FES & FOM & 3.5 & 8942 \\
\hline Solanum sanctaecatharinae Dunal & PI & FES & FOM & 2.3.5.7 & - \\
\hline \multicolumn{6}{|l|}{ STYRACACEAE } \\
\hline Styrax acuminatus Pohl & CL & - & FOM & 7 & 4375 \\
\hline Styrax leprosus Hook. \& Arn. & $\mathrm{CL}$ & - & FOM & 3.6 .7 & 191584 \\
\hline
\end{tabular}

${ }^{*}$ Exotic plants; PI - Pioneer; CL - Light-Demanding Climax; CS - Shade-Tolerant Climax; NC - Not classified; FES - Semi-deciduous Forest; FOM - Ombrophillous Mixed Forest. The species exclusively observed by Ziller (1998) do not provide information on occurrence in the plot groups and on the MBM registry. 
Table 1. Continued...

\begin{tabular}{|c|c|c|c|c|c|}
\hline Family/Species & GE & \multicolumn{2}{|c|}{$\begin{array}{l}\text { Vegetation } \\
\text { Formation }\end{array}$} & $\begin{array}{c}\text { Occurrence } \\
\text { in Plot } \\
\text { Groups }\end{array}$ & $\begin{array}{c}\text { Voucher in } \\
\text { the MBM }\end{array}$ \\
\hline \multicolumn{6}{|l|}{ SYMPLOCACEAE } \\
\hline Symplocos pentandra Occhioni & CL & FES & - & 7 & 23478 \\
\hline Symplocos uniflora (Pohl) Benth. & $\mathrm{CL}$ & - & FOM & - & - \\
\hline UNKNOWN & NC & - & - & 2.5.6.7 & - \\
\hline \multicolumn{6}{|l|}{ URTICACEAE } \\
\hline Cecropia pachystachya Trécul & PI & FES & - & 1.2.4.5.6 & 238741 \\
\hline Urera baccifera (L.) Gaudich. & PI & FES & FOM & 1.2.3.4.5.6 & 191567 \\
\hline \multicolumn{6}{|l|}{ VERBENACEAE } \\
\hline Aloysia virgata (Ruiz \& Pav.) Juss. & $\mathrm{CL}$ & FES & - & 1 & 261 \\
\hline Duranta vestita Cham. & PI & - & FOM & - & - \\
\hline \multicolumn{6}{|l|}{ WINTERACEAE } \\
\hline Drimys brasiliensis Miers & CS & - & FOM & - & - \\
\hline
\end{tabular}

${ }^{*}$ Exotic plants; PI - Pioneer; CL - Light-Demanding Climax; CS - Shade-Tolerant Climax; NC - Not classified; FES - Semi-deciduous Forest; FOM - Ombrophillous Mixed Forest. The species exclusively observed by Ziller (1998) do not provide information on occurrence in the plot groups and on the MBM registry.

group. For the same reason, six species could only be identified by Ziller (1998) at the genus level.

The most representative families in number of species were Fabaceae (34), Myrtaceae (18), and Lauraceae (16), followed by Euphorbiaceae, Meliaceae, Rutaceae, and Solanaceae with nine species each. The most frequent genera were Eugenia, Ocotea, and Solanum (seven), Nectandra (six), Inga, Lonchocarpus, and Trichilia (five), Casearia, Cordia, Ilex, and Zanthoxylum (four).

Considering only the sampling in the plots, the 10 species with the highest absolute density accounted for $44.96 \%$ of the total relative density: E. edulis (735), Sorocea bonplandii (241), Machaerium stipitatum (143), Nectandra megapotamica (137), Sebastiania brasiliensis, Cabralea canjerana and Ocotea diospyrifolia (134), Balfourodendron riedelianum (122), Chrysophyllum gonocarpum (104), and Syagrus romanzoffiana (92). The 10 species most commonly found in the plots and their respective frequencies (\%) were O. diospyrifolia (100), N. megapotamica (95.24), C. gonocarpum (95.24), S. romanzoffiana, Campomanesia xanthocarpa and Chrysophyllum marginatum (90.48), S. bonplandii, M. stipitatum and C. canjerana (85.71), and B. riedelianum (80.95).

Among the 218 species listed, 13 were not classified into ecological groups and vegetation formation because they were not identified at the species level or were exotic. Among the 205 remaining species, 78 (38.05\%) were classified as presenting FES characteristics, 17 (8.29\%) as FOM, and 110 (53.66\%) are of occurrence in both formations. Regarding successional stage, 70 species (34.15\%) were classified as Shade-Tolerant Climax, 92 (44.88\%) as Light-Demanding Climax, and 43 (20.98\%) as Pioneer.

Successional stage - In general, high values of richness and dominance were recorded in the plots and, in some cases, an expressive range of diameters and predominance of climax species were observed (Table 2). These results indicate that the forest remains well preserved.

In plot 6, low values of dominance and density were recorded $\left(22.02 \mathrm{~m}^{2} . \mathrm{ha}^{-1}\right.$ and 675 trees.ha-1), attributed to the high occurrence of Chusquea Kunth. (Criciúma) and Cyathea sp. (Xaxim-bravo), as well as to the presence of canopy gaps opened by the falling of large trees.

The highest dominance and density values were observed in plot 12, associated with the presence of Aspidosperma polyneuron - the largest diameter class, Light-Demanding Climax species (Apuleia leiocarpa, Cabralea canjerana, Diatenopteryx sorbifolia, and Ficus luschnathiana) - 70, 80 and 90 diameter classes, and the high density of E. edulis and S. bonplandii - the first diameter class. Such a physiognomy is typical of seasonal forests with low levels of human disturbance. 
Table 2. Relative frequency (\%) by diameter class, cover value for the ecological groups, and successional stage (SS) of plots installed in the Iguaçu National Park.

\begin{tabular}{|c|c|c|c|c|c|c|c|c|c|c|c|c|c|c|c|c|c|}
\hline \multirow{2}{*}{$\mathbf{P}$} & \multirow{2}{*}{$\mathbf{S}^{\prime}$} & \multirow{2}{*}{ DOA } & \multirow{2}{*}{$\mathbf{N}$} & \multicolumn{10}{|c|}{ Diameter Class (cm) } & \multicolumn{3}{|c|}{ Cover Value } & \multirow{2}{*}{ SS } \\
\hline & & & & 10 & 20 & 30 & 40 & 50 & 60 & 70 & 80 & 90 & $>95$ & PI & CL & CS & \\
\hline 1 & 47 & 25.23 & 805 & 64.60 & 18.01 & 10.56 & 4.35 & 1.86 & - & - & - & 0.62 & - & 10.12 & 89.95 & 94.77 & INT \\
\hline 2 & 45 & 33.10 & 805 & 64.60 & 12.42 & 8.70 & 5.5 & 4.35 & 3.73 & 0.62 & - & 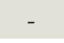 & - & 5.59 & 102.04 & 35.52 & $\mathrm{ADV}$ \\
\hline 3 & 39 & 33.16 & 100 & 73.64 & 11.36 & 7.73 & 3.18 & 2.73 & 0.91 & - & - & - & 0.45 & 4.36 & 57.69 & 125.83 & $\mathrm{ADV}$ \\
\hline 4 & 61 & 29.18 & 1,260 & 72.62 & 15.08 & 6.35 & 4.37 & 0.79 & 0.79 & - & - & - & - & 0.81 & 74.60 & 114.09 & $\mathrm{ADV}$ \\
\hline 5 & 44 & 29.90 & 720 & 59.72 & 19.44 & 9.03 & 7.64 & 1.39 & 0.69 & 0.69 & 0.69 & - & 0.69 & 9.40 & 97.74 & 92.00 & $\mathrm{ADV}$ \\
\hline 6 & 47 & 22.02 & 675 & 64.44 & 17.04 & 8.89 & 6.67 & 1.48 & 0.74 & 0.74 & - & - & 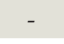 & 19.37 & 81.90 & 37 & NT \\
\hline 7 & 36 & 42.70 & 1,035 & 65.22 & 21.26 & 4.35 & 1.93 & 3.38 & 1.45 & 1.45 & - & 0.48 & 0.48 & 16.47 & 100.45 & 83.09 & $\mathrm{ADV}$ \\
\hline 8 & 45 & 37.04 & 785 & 58.60 & 21.66 & 8.28 & 5.10 & 2.55 & 1.91 & 0.64 & 0.64 & - & 0.64 & 34.57 & 76.81 & 88.63 & INT \\
\hline 9 & 42 & 27.13 & 795 & 67.92 & 17.61 & 3.77 & 4.4 & 2.52 & 3.14 & 0.63 & - & - & - & 08 & 106.32 & 0 & INT \\
\hline 10 & 49 & 38.03 & 855 & 76.02 & 9.36 & 5.85 & 3.5 & 0.58 & 2.34 & 1.17 & - & - & 1.17 & 18.64 & 8 & 99 & $\mathrm{ADV}$ \\
\hline 11 & 43 & 33.14 & ,510 & 84.77 & 7.62 & 3.64 & 0. & 2 & 0.33 & - & - & - & 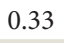 & 23 & 7 & 14 & DV \\
\hline 12 & 48 & 54.13 & 1,575 & 84.13 & 6.98 & 3.49 & 1.5 & 0.63 & 0.95 & 0.63 & 0.32 & 0.32 & 0.63 & 5.01 & 41.78 & 153 & $\mathrm{ADV}$ \\
\hline 13 & 59 & 29.09 & 960 & 77.08 & 9.90 & 3.13 & 5.21 & 2.60 & 0.52 & 1.04 & 0.52 & - & - & 32.17 & 79.38 & 82.13 & INT \\
\hline 14 & 49 & 23.65 & 730 & 74.66 & 9.59 & 8.22 & 1.37 & 3.42 & 2.05 & 0.68 & - & - & - & 41.79 & 90.24 & 67.21 & INT \\
\hline 15 & 48 & 38.73 & 755 & 70.20 & 9.93 & 10.60 & 2.65 & - & 3.97 & 0.66 & 0.66 & - & 1.32 & 11.62 & 77.26 & 110.02 & $\mathrm{ADV}$ \\
\hline 16 & 53 & 32.50 & 1,185 & 75.53 & 11.81 & 4.64 & 5.06 & 0.84 & 0.84 & 0.84 & - & 0.42 & - & 5.84 & 54.01 & 139.67 & $\mathrm{ADV}$ \\
\hline 17 & 45 & 39.13 & 1,110 & 75.68 & 8.11 & 7.21 & 1.80 & 4.05 & 2.25 & - & - & 0.45 & 0.45 & 5.58 & 71.83 & 122.58 & $\mathrm{ADV}$ \\
\hline 18 & 46 & 44.69 & 1,345 & 76.58 & 10.04 & 5.95 & 2.60 & 1.86 & 1.49 & 0.37 & - & 0.37 & 0.74 & 0.00 & 51.08 & 147.40 & $\mathrm{ADV}$ \\
\hline 19 & 52 & 37.13 & 1,500 & 72.33 & 16.00 & 6.33 & 2.33 & 1.67 & 1.00 & - & - & 0.33 & - & 11.38 & 138.18 & 49.12 & INT \\
\hline 20 & 45 & 24.84 & 785 & 68.15 & 14.01 & 5.73 & 8.28 & 3.82 & - & - & - & - & - & 9.69 & 121.49 & 68.06 & INT \\
\hline 21 & 47 & 23.71 & 1,205 & 71.37 & 19.92 & 6.22 & 1.24 & 1.24 & - & - & - & - & - & 21.05 & 100.39 & 78.08 & INT \\
\hline \multicolumn{4}{|c|}{ Average } & 72.67 & 13.24 & 6.26 & 3.49 & 2.02 & 1.28 & 0.44 & 0.12 & 0.16 & 0.3 & 14.94 & 83.15 & 100.53 & $\mathrm{ADV}$ \\
\hline
\end{tabular}

P - Plot; S' - Species richness; DOA - Dominance per hectare $\left(\mathrm{m}^{2} \cdot \mathrm{ha}^{-1}\right) ; \mathrm{N}$ - Density per hectare (trees.ha-1); Ecological group: PI - Pioneer, CL - Light-Demanding Climax, CS - Shade-Tolerant Climax; INT - Intermediate succession stage; ADV - Advanced succession stage.

The smaller diameter range observed in some plots suggested intermediate stages of succession. Hydromorphism was observed in the soil of plot 4, which limited the occurrence of large trees and justified its advanced successional classification. In plot 2, despite the limited range of diameter class $(70 \mathrm{~cm})$, the dominance value of $30 \mathrm{~m}^{2} \cdot \mathrm{ha}^{-1}$ indicated vegetation in good conservation condition. In plots 8 and 9, located on the bottom of a drainage slope, presence of Guadua chacoensis (Taquaruçu) contributed to the low density values and their classification in intermediate stages.

Preserved forests present points of morphological inversion distributed in different strata, reaching expressive heights. This characteristic could be observed in all plots and, despite the positive asymmetry and negative kurtosis, the distribution curves extended to heights $>13 \mathrm{~m}$, as shown in Table 3 . The highest relative frequencies were found below nine meters, justified by the high density in the initial diameter classes and recurrence of Shade-Tolerant Climax species.

Low frequency in the highest classes characterizes the emerging stratum above the relatively open canopy, typical of seasonal forests in southern Brazil (Leite \& Klein, 1990). The most prevalent species in these classes were A. polyneuron, A. leiocarpa, B. riedelianum, Ceiba speciosa, Cordia trichotoma, Jacaratia spinosa, M. stipitatum, Myrocarpus frondosus, and P. rigida.

Presence of Araucaria angustifolia above $19 \mathrm{~m}$ was observed in plots 19,20 , and 21 . This species is associated with $P$. rigida, Casearia decandra, and Nectandra lanceolata between 11 and $17 \mathrm{~m}$, and the high density of C. canjerana, C. xanthocarpa, and Ilex paraguariensis in the understory, between 3 and $7 \mathrm{~m}$, characterized the vertical structure of this transitional vegetation between FOM and FES. Specimens of emergent species in FES were identified within these plots in the classes of 7, 9, and $11 \mathrm{~m}$, including A. polyneuron, C. trichotoma, and M. frondosus. 
Table 3. Relative frequency (\%) by height class to the morphological inversion point for the 21 plots installed in the Iguaçu National Park.

\begin{tabular}{|c|c|c|c|c|c|c|c|c|c|c|c|c|c|c|}
\hline \multirow[t]{2}{*}{ Plot } & \multirow[t]{2}{*}{$\mathbf{N}$} & \multicolumn{11}{|c|}{ Center of height class from ground to the morphological inversion } & \multirow[t]{2}{*}{ Asymmetry } & \multirow[t]{2}{*}{ Kurtosis } \\
\hline & & 1 & 3 & 5 & 7 & 9 & 11 & 13 & 15 & 17 & 19 & 21 & & \\
\hline 1 & 805 & 4.35 & 36.02 & 31.06 & 16.77 & 3.73 & 4.97 & 0.62 & 1.86 & - & 0.62 & - & 1.45 & 0.79 \\
\hline 2 & 805 & 5.59 & 36.02 & 28.57 & 13.66 & 8.70 & 2.48 & 1.86 & 2.48 & 0.62 & - & - & 1.42 & 0.68 \\
\hline 3 & 1,100 & 4.09 & 27.27 & 24.09 & 16.36 & 10.00 & 8.64 & 5.00 & 2.27 & 1.36 & 0.91 & - & 1.09 & -0.07 \\
\hline 4 & 1,260 & 5.95 & 28.97 & 34.13 & 17.06 & 5.16 & 5.16 & 1.59 & 1.19 & 0.79 & - & - & 1.23 & 0.00 \\
\hline 5 & 720 & 5.56 & 22.92 & 22.92 & 17.36 & 13.19 & 9.03 & 6.25 & 0.69 & - & 1.39 & 0.69 & 0.41 & -1.39 \\
\hline 6 & 675 & 4.44 & 28.89 & 29.63 & 18.52 & 10.37 & 2.96 & 1.48 & 0.74 & 1.48 & 1.48 & - & 1.18 & -0.06 \\
\hline 7 & 1,035 & 2.42 & 23.19 & 29.95 & 22.71 & 14.98 & 3.38 & 1.93 & - & 0.97 & 0.48 & - & 0.96 & -0.57 \\
\hline 8 & 785 & 6.37 & 19.11 & 24.20 & 19.75 & 10.19 & 10.83 & 6.37 & 2.55 & 0.64 & - & - & .66 & -0.95 \\
\hline 9 & 795 & 2.52 & 25.79 & 25.79 & 18.87 & 15.72 & 6.92 & 3.77 & 0.63 & - & - & - & 0.87 & -0.84 \\
\hline 10 & 855 & 4.09 & 24.56 & 31.58 & 19.30 & 10.53 & 4.68 & 2.92 & 0.58 & 1.17 & 0.58 & - & 0.97 & -0.81 \\
\hline 11 & 1,510 & 2.65 & 16.56 & 34.11 & 22.85 & 12.91 & 8.61 & 1.66 & 0.66 & - & - & - & 1.07 & 0.06 \\
\hline 12 & 1,575 & 1.27 & 14.29 & 24.76 & 21.59 & 13.97 & 12.06 & 4.44 & 3.17 & 2.86 & 0.63 & 0.95 & 0.81 & -0.37 \\
\hline 13 & 960 & 4.69 & 23.44 & 34.90 & 19.27 & 11.98 & 3.65 & 1.56 & - & - & 0.52 & - & 1.31 & 0.62 \\
\hline 14 & 730 & 4.79 & 27.40 & 32.19 & 20.55 & 8.90 & 2.05 & 1.37 & 1.37 & 0.68 & - & 0.68 & 1.25 & 0.00 \\
\hline 15 & 755 & 1.32 & 20.53 & 18.54 & 23.84 & 19.87 & 9.27 & 3.31 & 2.65 & 0.66 & - & - & 0.51 & -1.79 \\
\hline 16 & 1,185 & 2.11 & 20.68 & 24.47 & 16.03 & 11.39 & 8.44 & 10.97 & 2.53 & 1.27 & 1.69 & 0.42 & 0.72 & -0.91 \\
\hline 17 & 1,110 & 1.80 & 18.47 & 28.83 & 13.06 & 15.32 & 7.66 & 10.36 & 3.15 & 1.35 & - & - & 1.15 & 1.37 \\
\hline 18 & 1,345 & 1.12 & 15.61 & 30.11 & 14.50 & 10.04 & 15.99 & 7.43 & 4.09 & 0.74 & - & 0.37 & 1.13 & 1.24 \\
\hline 19 & 1,500 & 3.00 & 15.33 & 26.00 & 25.67 & 15.67 & 9.00 & 2.67 & 1.00 & 1.00 & 0.67 & - & 1.00 & -0.37 \\
\hline 20 & 785 & 3.82 & 27.39 & 21.02 & 24.20 & 14.01 & 5.10 & 4.46 & - & - & - & - & 0.89 & -0.93 \\
\hline 21 & 1,205 & 4.15 & 19.92 & 33.61 & 24.48 & 13.69 & 2.90 & 0.83 & 0.41 & - & - & - & 1.17 & 0.01 \\
\hline
\end{tabular}

$\mathrm{N}$ - Density per hectare (trees.ha-1).

\section{DISCUSSION}

Floristic composition - Out of the 218 tree species listed, 51 were exclusive of the survey by Ziller (1998), 86 were exclusive of this survey, and 81 were common to both surveys. Ramos et al. (2008) identified 238 species in a FES remnant in Sao Paulo state and Silva \& Soares-Silva (2000) identified 206 species in a FES in northern Parana state; Gasper et al. (2013b), identified 233 species between trees and shrubs in a Deciduous Forest in Santa Catarina state. Other researchers reported lower species richness in surveys conducted in smaller FES fragments in southern Brazil: Jarenkow \& Waechter (2001), Giehl \& Jarenkow (2008), Scipioni et al. (2011), Ríos et al. (2010), and Bianchini et al. (2003) identified 55, 82, 72, 64 and 116 species, respectively.

In this research, the botanical families Fabaceae and Myrtaceae were the most representative in number of species, corroborating the studies by Oliveira-Filho \& Fontes (2000) in an FES in southeastern Brazil and
Jarenkow \& Waechter (2001) in the central region of Rio Grande do Sul state. These families have also presented higher richness in surveys conducted in northern Parana state (Silva \& Soares-Silva, 2000), northwestern Santa Catarina state (Scipioni et al., 2011), Rio Grande do Sul state (Giehl \& Jarenkow, 2008), and in northeastern Argentina (Ríos et al., 2010).

Other families common to the INP also reported by Jarenkow \& Waechter (2001), Silva \& Soares-Silva (2000), and Ríos et al. (2010) include Lauraceae and Meliaceae, observed among the five richest families. In contrast, Oliveira-Filho \& Fontes (2000) observed high species richness only for Lauraceae. Scipioni et al. (2011) and Giehl \& Jarenkow (2008) reported richness only for Meliaceae, associated with early succession in the former study and with alluvial forest in the latter.

Meira \& Martins (2002) performed a comparative analysis of similarity between fragments of montane FES in Minas Gerais state (between 650 and $800 \mathrm{~m}$ asl) and semideciduous forests in Sao Paulo and northern Parana states. Based on the results, the authors hypothesized 
that the floristic similarity between montane and submontane FES increases proportionally to latitude.

Comparison between the species that occurred in plots located in the submontane FES of the INP (between 100 and $600 \mathrm{~m}$ asl) identified 27 species in common with the study by Meira \& Martins (2002), apparently confirming their hypothesis. Some species even presented high density and dominance values, namely, C. gonocarpum, M. stipitatum, and S. bonplandii.

Also in support of the hypothesis of the aforementioned authors, high amplitude of dispersion along the altitudinal gradient was found for 52 seasonal species in the INP. These species are altitude indicators in the state of Sao Paulo, as described by Meira et al. (1989). Results of this analysis revealed 13 species occurring in the INP, 11 of which found in submontane FES: Alchornea triplinervia, Luehea divaricata, C. canjerana, Cedrela fissilis, C. decandra, Casearia obliqua, Allophylus edulis, C. speciosa, Handroanthus albus, Myrsine umbellata, and Pisonia ambigua. Cupania vernalis occurred only in montane regions $600 \mathrm{~m}$ asl, whereas Roupala brasiliensis was restricted to an ecotone between FES and FOM, $700 \mathrm{~m}$ asl.

Also corroborating this result, floristic similarity was observed between the montane FES (600-700 m asl) and the submontane Decidual Forests at higher latitudes below $550 \mathrm{~m}$ asl, as described by Jarenkow \& Waechter (2001) and Scipioni et al. (2011). Those studies found a total of 55 and 79 species, respectively, of which $30(54.54 \%)$ and $42(53.16 \%)$ were common to those of the present study.

Successional stage - Budowski (1965) reported that in dense undisturbed forests or in forests in more advanced successional stages, the recruitment of Pioneer species is subject to emergence of canopy gaps, which may explain the low cover value for this ecological group in the INP. Holz et al. (2009) reported that the native forests of northeastern Argentina were mostly composed of Light-Demanding Climax and Shade-Tolerant Climax species, whereas Pioneer species accounted for $25 \%$.

Shade-Tolerant Climax species are also widely recurrent and represented in greater abundance by E. edulis, S. bonplandii, Sebastiania brasiliensis, N. megapotamica, O. diospyrifolia, B. riedelianum, and $C$. gonocarpum, also in agreement with the results found by Holz et al. (2009). In the INP, this ecological group amounted to $100.53 \%$ of the total cover value for vegetation and, together with the Light-Demanding Climax species, to $183.68 \%$.

Ziller (1998) described the central region of the Park as showing fewer traces of anthropogenic activities, illustrated by the lush vegetation and high floristic diversity. Furthermore, in addition to A. polyneuron, other species characteristic of vegetation in advanced-stage seasonal forests were recurrent in this region, including A. leiocarpa, $M$. frondosus, $B$. riedelianum, Jacaratia spinosa, Lonchocarpus muehlbergianus, and Holocalyx balansae. Also noteworthy is the wide range of diameters observed in the plots established in that region (plots 7 to 18 ).

However, some of the plots located on the slopes of the river valley of the central region showed a narrower range of diameters and recurrence of Pioneer species at intermediate stages of ecological succession. This finding may be associated with the rugged terrain and increased water availability (Muchailh et al., 2010). Another related factor may be the increased light incidence in the understory of the plots located on the drainage slopes oriented to the East, resulting in an edge effect (Schorn \& Galvão 2009).

The narrower range of diameters and the high concentration of trees with morphological inversion point $<7 \mathrm{~m}$ indicate intermediate successional stages for two plots in the southern and southwestern parts of the INP (plots 1 and 6). Ziller (1998) pointed out that, unlike the logging that occurred in other regions, the anthropogenic activities in this region included clearing of vegetation for agricultural use, which slowed the restoration process to its original state.

Further North in the Park, in the transition zone between Semideciduous and Ombrophillous Forests, ecological succession proceeds at an intermediate stage, indicated by reduction in dominance, lower range of diameters, and lower morphological inversion point. This result can be explained by the high level of anthropogenic disturbance because of the forest proximity to the municipality of Santa Tereza do Oeste (Ziller, 1998). Despite the intensified logging activities occurred in this area, the vegetation was not completely removed, and thus maintained its potential for recovery. Evidence of this potential is observed in the presence of species of high commercial value typical of Ombrophillous Forests. 


\section{CONCLUSIONS}

In general, forests in advanced successional stage were observed throughout the Iguaçu National Park (INP). The central region presents few characteristics indicative of anthropogenic activities and portrays, more accurately, the original seasonal forests that occurred in the Parana River basin. The forests of the South and far North areas of the INP still present signs of anthropogenic activities, where some species show low recurrence and depend on a long period of in disturbance and isolation to return to its original state.

Evidence of the effect of altitude and latitude on the distribution of species of seasonal forests was observed to compare the results of this survey with those of studies conducted in the Southeast and extreme South regions of Brazil.

\section{ACKNOWLEDGEMENTS}

The authors are grateful to Chico Mendes Institute for Biodiversity Conservation (ICMBio) for the authorization and availability of the physical structure to conduct this study, Coordination for the Improvement of Higher Education Personnel (CAPES) for the financial support in the form of a scholarship, and to National Council for Scientific and Technological Development (CNPQ) for the financial assistance to conduct the fieldwork.

\section{SUBMISSION STATUS}

Received: 15 dec., 2015

Accepted: 25 may, 2018

\section{CORRESPONDENCE TO}

\section{Ronan Felipe Souza}

Laboratório de Dendrometria, Departamento de Engenharia Florestal, Universidade Federal do Paraná - UFPR, Av. Lothário Meissner, 632, CEP 80210-170, Curitiba, PR, Brasil e-mail: ronanflorestal@gmail.com

\section{FINANCIAL SUPPORT}

This study was funded by National Council for Scientific and Technological Development (CNPq), grant no. 484747/2011-8.

\section{REFERENCES}

Alvares CA, Stape JL, Sentelhas PC, Gonçalves JLM, Sparovek G. Köppen's climate classification map for Brazil. Meteorologische Zeitschrift 2013; 22(6): 711-728. http:// dx.doi.org/10.1127/0941-2948/2013/0507.

Avila AL, Araujo MM, Longhi SJ, Gasparin E. Agrupamentos florísticos na regeneração natural em remanescente de Floresta Ombrófila Mista, RS, Brasil. Scientia Forestalis 2011; 39(91): 331-342.

Bhering SB. Mapa de solos do Estado do Paraná: Escala 1:250.000. Rio de Janeiro: Embrapa Solos; 2007.

Bianchini E, Popolo RS, Dias MC, Pimenta JA. Diversity and structure of a tree species community in flooded area in the municipality of Londrina, Southern Brazil. Acta Botanica Brasílica 2003; 17(3): 405-419. http://dx.doi. org/10.1590/S0102-33062003000300008.

Brasil. Resolução Conama n. 2, 18 de Março de 1994. Diário Oficial da República Federativa do Brasil, Brasília, DF (1994 mar).

Budowski G. Distribution of tropical american rain forest species in the light of successional processes. Turrialba 1965; 15(1): 40-42.

Felfili JM, Eisenlohr PV, Melo MMRF, Andrade LA, Meira JAA No. Fitossociologia no Brasil: métodos e estudos de casos. Viçosa: Editora UFV; 2011.

Ferreira LM. Revisão do plano de manejo do Parque Nacional do Iguaçu encarte 5, com vistas à revisão do plano de manejo. Brasília: IBAMA: FUPEF; 1999.

Gasper AL, Sevegnani L, Vibrans AC, Sobral M, Uhlman A, Lingner DV et al. Flora of the mixed ombrophyllous forest in Santa Catarina state, according of the forest and floristic inventory of Santa Catarina. Rodriguésia 2013a; 64(2): 201-210. http://dx.doi.org/10.1590/S217578602013000200001 .

Gasper AL, Uhlman A, Sevegnani L, Lingner DV, RigonJúnior MJ, Verdi M et al. Floristic and forest inventory of santa catarina: species of seasonal deciduous forests. Rodriguésia 2013b; 64(3): 427-443. http://dx.doi.org/10.1590/ S2175-78602013000300001.

Giehl ELH, Jarenkow JA. Structural gradient of the tree component and relationship with flooding in a riverine forest, Rio Uruguai, southern Brazil. Acta Botanica Brasílica 2008; 22(3): 741-753. http://dx.doi.org/10.1590/ S0102-33062008000300012.

Holz S, Placci GP, Quintana RD. Effects of History of use on secondary forest regeneration in the Upper Parana Atlantic Forest (Misiones, Argentina). Forest Ecology and Management 2009; 258(7): 1629-1642. http://dx.doi. org/10.1016/j.foreco.2009.07.023

Jarenkow JA, Waechter JL. Composition, structure and floristic relations of the tree component of a seasonal 
forest in Rio Grande do Sul, Brazil. Brazilian Journal of Botany 2001; 24(3): 263-272.

Leite PF, Klein RM. Vegetação. In: Instituto Brasileiro de Geografia e Estatística - IBGE. Geografia do Brasil: região sul. Rio de Janeiro: IBGE; 1990.

Lin G, Stralberg D, Gong G, Huang Z, Ye W, Wu L. Separating the effects of environment and space on tree species distribution: from population to community. PLoS One 2013; 8(2): e56171. http://dx.doi.org/10.1371/ journal.pone.0056171. PMid:23409151.

Meira JAA No, Bernacci LC, Grombone MT, Tamashiro JY, Leitão HF Fo. Floristic composition of the semideciduous mountain forest of the Grota Funda Municipal Park, Atibaia, São Paulo. Acta Botanica Brasílica 1989; 3(2): 5174. http://dx.doi.org/10.1590/S0102-33061989000200006.

Meira JAA No, Martins FR. Floristic composition of a montane seasonal semideciduous tropical forest in Viçosa MG Brasil. Revista Árvore 2002; 26(4): 437-446.

Muchailh MC, Roderjan CV, Campos JB, Machado ALT, Curcio GR. Methodology for planning fragmented landscapes aiming the creation of Ecological Corridors. Floresta 2010; 40(1): 147-162.

Mueller-Dambois D, Ellenberg H. Aims and methods of vegetation ecology. New York: John Wiley e Sons; 1974.

Oliveira-Filho AT, Fontes MA. Patterns of floristic differentiation among atlantic forest in southeastern Brazil and the influence of climate. Biotropica 2000; 32(4b): 793 810. http://dx.doi.org/10.1111/j.1744-7429.2000.tb00619.x.

Oliveira-Filho AT, Vilela EA, Carvalho DA, Gavilanes ML. Effects of soils and topography on the distribution of tree species in a tropical riverine forest in south-eastern Brasil. Journal of Tropical Ecology 1994; 10(4): 483-508. https://doi.org/10.1017/S0266467400008178.

Ramos VS, Durigan G, Franco GADC, Siqueira MF, Rodrigues RR. Árvores da floresta estacional semidecidual: guia de identificação de espécies. São Paulo: Edusp; 2008.

Ríos RC, Galvão F, Curcio GR. Structure of main arboreal species in cruce caballero park and its floristic similarity with areas from Argentina and Brazil. Ciência Florestal 2010; 20(2): 193-206

Schorn LA, Galvão F. Dynamics of arboreal strate in three successional stages of a fragment of the atlantic rain forest in Blumenau, SC. Cerne 2009; 15(2): 221-235.

Scipioni MC, Finger CAG, Cantarelli EB, Denardi L, Meyer EA. Phytosociological study in a forest fragment in the northwest of Rio Grande do Sul state. Ciência Florestal 2011; 21(3): 407-417. http://dx.doi.org/10.5902/198050983799.

Silva CPC, Oliveira-Filho AT, Van Den Berg E, Scolforo JR, Mello JM, Oliveira AD. Composição florística na floresta estacional semidecidual e floresta ombrófila. In: Scolforo JR, editor. Inventário florestal de minas gerais: floresta estacional semidecidual e ombrófila - florística, estrutura, diversidade, similaridade, distribuição diamétrica e de altura, volumetria, tendências de crescimento e áreas aptas para o manejo florestal. 3rd ed. Lavras: UFLA; 2008.

Silva FC, Soares-Silva LH. Arboreal flora of the Godoy Forest State Park, Londrina, PR, Brazil. Edinburgh Journal of Botany 2000; 57(1): 107-120. http://dx.doi.org/10.1017/ S096042860000007X.

Souza RF, Machado SA, Galvão F, Figueiredo A Fo. Fitossociologia da vegetação arbórea do Parque Nacional do Iguaçu. Ciência Florestal 2017; 27(3): 853-869. http:// dx.doi.org/10.5902/1980509828635.

Swaine MD, Whitmore TC. On the definition of ecological species groups in tropical rain forests. Vegetatio 1988; 75(1-2): 81-86. http://dx.doi.org/10.1007/BF00044629.

The Angiosperm Phylogeny Group - APG III. An update of the Angiosperm Phylogeny Group classification for the orders and families of flowering plants: APG III. Botanical Journal of the Linnean Society 2009; 161(2): 105-121. http:// dx.doi.org/10.1111/j.1095-8339.2009.00996.x.

Whitmore TC. Canopy gaps and the two major groups of forest trees. Ecology 1989; 70(3): 536-538. http://dx.doi. org/10.2307/1940195.

Ziller SR. Avaliação ecológica rápida do Parque Nacional do Iguaçu. Curitiba: IBAMA; 1998. 